\title{
Short communication: The role of genotypes from animals without phenotypes in single-step genomic evaluations
}

\author{
T. Shabalina, ${ }^{1}$ E. C. G. Pimentel, ${ }^{1,2}$ C. Edel, L. Plieschke, R. Emmerling, and K.-U. Götz \\ Institute of Animal Breeding, Bavarian State Research Center for Agriculture, 85586 Grub, Germany
}

\begin{abstract}
In a 2-step genomic system, genotypes of animals without phenotypes do not influence genomic prediction of other animals, but that might not be the case in single-step systems. We investigated the effects of including genotypes from culled bulls on the reliability of genomic predictions from single-step evaluations. Four scenarios with a constant amount of phenotypic information and increasing numbers of genotypes from culled bulls were simulated and compared with respect to prediction reliability. With increasing numbers of genotyped culled bulls, there was a corresponding increase in prediction reliability. For instance, in our simulation scenario the reliability for selection candidates was twice as large when all culled bulls from the last 4 generations were included in the analysis. Single-step evaluations imply the imputation of all nongenotyped animals in the pedigree. We showed that this imputation was increasingly more accurate as increasingly more genotypic information from the culled bulls was taken into account. This resulted in higher prediction reliabilities. The extent of the benefit from including genotypes from culled bulls might be more relevant for small populations with low levels of reliabilities.
\end{abstract}

Key words: genomic breeding value, imputation, numerator relationship, SNP

\section{Short Communication}

The genomic selection method proposed by Meuwissen et al. (2001) consisted of a calibration step using information from genotyped animals with phenotypes and a prediction step for genotyped animals without phenotypes. This 2-step model can be formulated as a function of marker effects or of animal effects, with covariance structure described by the genomic relationship matrix G (VanRaden, 2008). The latter has been usually referred to as genomic BLUP. Legarra

\footnotetext{
Received February 15, 2017.

Accepted June 15, 2017.

${ }^{1}$ These authors contributed equally to this manuscript.

${ }^{2}$ Corresponding author: Eduardo.Pimentel@LfL.bayern.de
}

et al. (2009) and Christensen and Lund (2010) proposed a single-step model [single-step genomic BLUP (ssGBLUP)] for genomic evaluation in which all available information from genotypes, phenotypes, and pedigree is simultaneously used. This single-step model is expected to provide unbiased predictions even with selection and nonrandom mating (Fernando et al., 2014).

Due to decreasing genotyping costs, the number of animals being genotyped has been constantly increasing, which can lead to some computational challenges. This may be an issue, in particular, in single-step evaluations in which the system sizes are at least as large as the number of animals in the pedigree. Attempts to overcome these challenges have been proposed. Misztal et al. (2014) introduced a method for approximating the inverse of $\mathbf{G}$, which is needed in ssGBLUP. Lourenco et al. (2014) examined the effect of reducing the number of generations used in ssGBLUP and reported that truncating old data may reduce computational requirements without decreasing reliability of prediction.

In a 2-step model, genotyped animals without phenotypes do not take part in the calibration step and, therefore, have no influence on predicted breeding values of other animals. In practice, this would mean that once young genotyped bulls have been culled their genotypes can be neglected in further runs of a 2-step genomic evaluation system. If such culled genotyped animals can also be neglected in ssGBLUP without affecting the prediction reliability for other animals, this would help reducing computational costs. However, Legarra et al. (2014) suggested that such animals should not be eliminated in ssGBLUP because they may change pedigree relationships across other animals. The objective of the present study was to investigate whether, to which extent, and how genotyped animals without phenotypes can influence the reliability of prediction in ssGBLUP.

To investigate these questions we simulated data using the software QMSim (Sargolzaei and Schenkel, 2009). Simulation was done in a way to mimic the structure and linkage disequilibrium found in the German-Austrian Fleckvieh population, as described in Plieschke et al. (2016). Briefly, a historical population of 2,000 unrelated animals with equal sex ratio was 
Table 1. Description of the amount of genotypic information in the 4 scenarios

\begin{tabular}{lcccc}
\hline & \multicolumn{3}{c}{ Number of genotyped males } \\
\cline { 2 - 5 } Generation & Scenario 1 & Scenario 2 & Scenario 3 & Scenario $4^{1}$ \\
\hline 5 & 1,050 & 1,050 & 15,000 & $1,050+1,050$ \\
6 & 1,050 & 1,050 & 15,000 & $1,050+1,050$ \\
7 & 1,050 & 1,050 & 15,000 & $1,050+1,050$ \\
8 & 1,050 & 15,000 & 15,000 & $1,050+1,050$ \\
9 & 3,000 & 3,000 & 3,000 & 3,000 \\
Total & 7,200 & 21,150 & 63,000 & 1,400 \\
\hline
\end{tabular}

${ }^{1}$ The 1,050 highest and 1,050 lowest ranking bulls from generations 5 to 8 .

generated and randomly mated for 2,500 generations. To create a linkage disequilibrium structure similar to the one observed in Fleckvieh, a bottleneck was introduced in generation 2,501 by reducing the number of breeding animals to 150, which approximately corresponds to the effective population size estimated for Fleckvieh (Pausch et al., 2013). Afterward the number of breeding animals was increased to 30,000 dams and 1,500 sires. These were the founder animals (generation 0 ) of the recent population, which was propagated for another 9 generations. In each of them, 15,000 female and 15,000 male offspring were produced. Generations were overlapping and in every generation $30 \%$ of the dams $(\mathrm{n}=9,000)$ and $70 \%$ of the sires $(\mathrm{n}=1,050)$ were replaced. These replacement ratios are quite similar to the situation observed in the real Fleckvieh population. Breeding animals were selected based on their EBV, which were calculated by QMSim. The reliabilities of these EBV were approximately 0.6, which resulted from the availability of information on parent average plus a single phenotypic record of the own animal. The simulated genome consisted of 30 chromosomes with 100 centimorgans. Single nucleotide polymorphisms were evenly distributed and QTL were randomly distributed across the genome $(49,800 \mathrm{SNP}$ and 900 QTL in total). To ensure sufficient quality of the genotypic data, SNP that significantly deviated from Hardy-Weinberg equilibrium $\left(P<10^{-5}\right)$ and SNP with minor allele frequency lower than 0.02 were excluded. After quality control, about 37,500 SNP and 700 QTL remained in the data set. True breeding values were simulated as the sum of QTL effects, and phenotypes were generated considering a heritability of 0.4 . The polygenic nature of the trait was ensured by the relatively high number of QTL whose effects were drawn from a uniform distribution; we did this to prevent the occurrence of a few isolated large QTL effects. Despite the fact that QMSim simulated phenotypes for all animals, we only used phenotypes of females to depict a situation observed in dairy cattle. Simulation was replicated 5 times and we also ran one repetition in which replacement animals were selected at random.
To show the influence of genotypes without phenotypes, we assigned the simulated data to 4 different scenarios containing an increasing number of culled genotyped males. Phenotypic information did not change across scenarios and consisted of phenotypes of the cows from generations 3 to $8(15,000$ cows with phenotypes per generation, summing to a total of 90,000 phenotypic records). The numbers of genotyped male animals from each generation for the 4 different scenarios are shown in Table 1.

The first scenario (S1) contained genotypes of all the bulls with daughter performance from generations 5 to 7 , of the top young bulls considered being selected but still without daughter performance from generation 8 , and of the young selection candidates from generation 9. The amount of genotypic information in this reference scenario depicts a typical situation in dairy genomic programs running 2-step evaluations, in which only genotypes of calibration bulls, genomic young bulls, and candidates are used. The second scenario (S2) additionally contained genotypes of all culled young bulls from generation 8 . In the third scenario (S3), all culled bulls from generations 5 to 7 were also included. The fourth scenario (S4) was an alternative to S3 in which not all, but only the subset of the highest and lowest ranking bulls from generations 5 to 8 were included. This was done to check if the addition of the lowest-ranking nonselected animals would make the additional genotype pool representative enough so that at least some computational demands could be saved.

We estimated genomic EBV for all scenarios with ssGBLUP using the software package MiX99 Release VIII/2015 (Lidauer et al., 2015) for solving the mixed model equations. The statistical model included a random animal effect and an overall mean as fixed effect. The system of equations to solve is similar to Henderson's mixed model equations for an animal model, except that the covariance structure of the animal effect is described by a matrix $\mathbf{H}$ instead of the usual numerator relationship matrix A. According to Aguilar et al. (2010) and Christensen and Lund (2010), the inverse of $\mathbf{H}$ has the following form: 


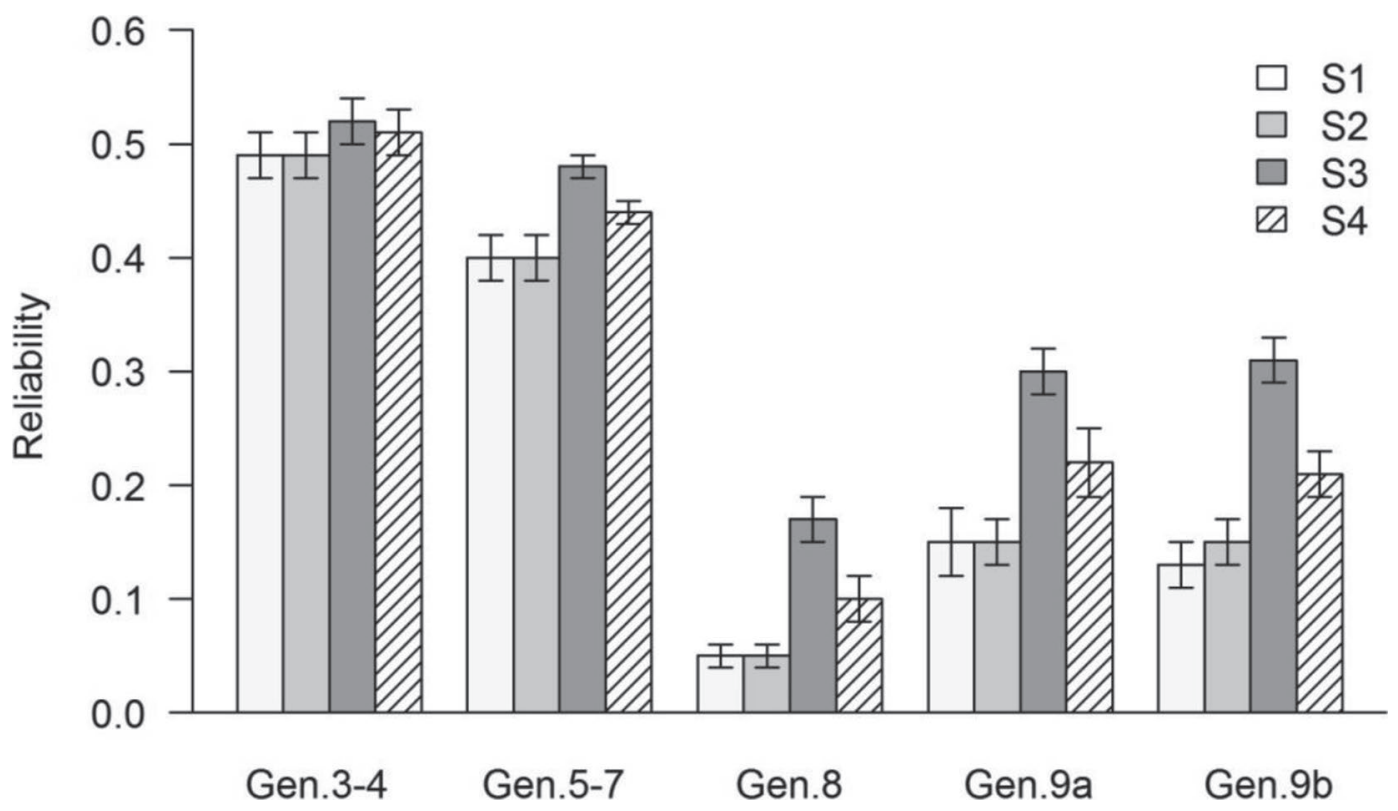

Figure 1. Average reliability $( \pm \mathrm{SD})$ for different groups of bulls in the different scenarios $(\mathrm{S})$. Gen.3-4 = nongenotyped sires; Gen.5-7 = genotyped sires with daughter performance; Gen.8 = young genotyped bulls without daughter performance; Gen.9a = young genotyped selection candidates (sons of sires from Gen.5-7); Gen.9b = young genotyped selection candidates (sons of sires from Gen.8).

$$
\mathbf{H}^{-1}=\mathbf{A}^{-1}+\left[\begin{array}{cc}
0 & 0 \\
0 & \mathbf{G}^{-1}-\mathbf{A}_{22}^{-1}
\end{array}\right],
$$

where $\mathbf{A}_{22}$ is the numerator relationship matrix for genotyped animals. The block $\left[\mathbf{G}^{-1}-\mathbf{A}_{22}^{-1}\right]$ was provided as input to MiX99. Matrix $\mathbf{G}$ was computed according to the first method of VanRaden (2008), using the simulated true base allele frequencies. For numerical stability, we blended $\mathbf{G}$ with $1 \%$ of $\mathbf{A}$.

Reliabilities of estimated breeding values for the different scenarios were calculated as the coefficient of determination (adjusted $\mathrm{R}^{2}$ ) of the regression of true breeding values on genomic EBV. For each scenario, we present the average reliability over the 5 simulated replicates.

Average reliabilities observed in the different scenarios are shown in Figure 1 for sires from generations 3 to 8 and male selection candidates from generation 9. Results from generation 9 are further divided into group 9a (sons of proven bulls from generations 5 to 7 ) and group 9b (sons of genomic young bulls from generation 8). In general, reliabilities increased with additional genotypic information. Differences between $\mathrm{S} 1$ and $\mathrm{S} 2$ were negligible in most generations. The highest reliabilities were achieved in S3 and reliabilities from S4 were higher than from S1 and S2, but not as large as from S3.

Reliabilities of sires in generations 3 to 4 and 5 to 7 were generally higher, as they already included informa- tion from daughter performances; this is also the reason why additional genotypes led to a smaller increase in reliability for these proven sires. Reliabilities of young bulls from generations 8 and 9 depended completely on parent average and genomic information, and therefore profited most from additional genotypes. The average reliability of young bulls from generation 8 was 0.17 in S3 and 0.05 in S1 and S2. These young bulls are a selected sample of the male animals from generation 8 , which explains the low reliability level in comparison with the unselected candidates from generation 9. For the selection candidates in generation 9 , we observed an increase from around 0.15 in S1 and S2 to around 0.31 in S3. Differences between groups 9a and 9b were small. The average reliabilities in S4 were around 60 to $70 \%$ of the reliabilities in S3 for generation 8 and 9 . Similar trends across scenarios were also observed in the simulation done with random selection (data not shown).

As mentioned, the amount of phenotypic information across scenarios was exactly the same. Therefore, the only source of additional information contributing to the observed improvement in reliabilities must have been the genotypes from culled males. Let us recall the form of the $\mathbf{H}$ matrix, described by Legarra et al. (2009) as

$$
\mathbf{H}=\left[\begin{array}{cc}
\mathbf{A}_{11}-\mathbf{A}_{12} \mathbf{A}_{22}^{-1} \mathbf{A}_{21}+\mathbf{A}_{12} \mathbf{A}_{22}^{-1} \mathbf{G A}_{22}^{-1} \mathbf{A}_{21} & \mathbf{A}_{12} \mathbf{A}_{22}^{-1} \mathbf{G} \\
\mathbf{G A}_{22}^{-1} \mathbf{A}_{21} & \mathbf{G}
\end{array}\right],
$$


where subscript 1 stands for the group of nongenotyped animals and 2 for the group of genotyped animals. From the above formula it is clear that the $\mathbf{G}$ matrix changes the elements in the blocks of $\mathbf{H}$ that correspond to nongenotyped animals. This transmission of information from the 22 block to the other blocks of $\mathbf{H}$ can be equivalently interpreted as imputation, as shown by Fernando et al. (2014). Therefore, one should expect that an increase in the amount of information in $\mathbf{G}$ would improve the imputation quality of the nongenotyped animals.

To illustrate the effects of growing information contents in $\mathbf{G}$ on the imputation quality of the nongenotyped animals, we explicitly imputed genotypes of the sires in generation 3 and 4 and compared them with their true genotypes; the imputation was done using the formula presented by Fernando et al. (2014). By adding genotypes of culled bulls, we expected that the number of genotyped sons of bulls from generation 3 and 4 increased, so that more information is available for imputing their genotypes. Table 2 shows the average number of sons and the correlation between imputed and true genotypes of the sires in generations 3 and 4 , as well as the expected imputation accuracy from progeny information calculated using selection index theory (Lush, 1931). The equation used for calculating the expected accuracy was

$$
\sqrt{\frac{n h^{2}}{4+(n-1) h^{2}}},
$$

where $\mathrm{n}$ is the number of progeny and $\mathrm{h}^{2}$ is the heritability, which was assumed to be 0.99 to account for a small proportion of genotyping errors.

With increasing numbers of genotypes from culled bulls in the different scenarios, we noted a corresponding increase in the average number of genotyped sons per sire, especially in S3, where all genotypes from culled bulls were used. This increase resulted in higher correlations between imputed and true genotypes. We also found good agreement between the tendencies of computed correlations and expected imputation accuracies based on the number of sons. One should take into account that these expected values are rather conserva- tive, because only information from direct offspring was used in the calculations.

Improvements in imputation accuracy result in increased prediction reliability. In the case of the bulls from generations 3 and 4, one can directly see this connection because they have daughters with records in generations 4 to 8 . Imputing the genotypes of these bulls means expanding the set of animals contributing both genotypic and phenotypic information to the system. Thinking in terms of 2-step models, it would mean enlarging the calibration set through imputation of ungenotyped bulls, as investigated by Pszczola et al. (2011). The extent to which this enlargement may be beneficial is determined by how well the imputation is done. Close relationships between the genotyped and ungenotyped animals, and especially the availability of Mendelian sampling information, considerably improve imputation accuracy, as shown by Pszczola et al. (2012), which the observed and expected imputation accuracies presented in Table 2 confirm.

The additional information in $\mathbf{G}$ actually replicates over the whole $\mathbf{H}$ matrix, influencing imputation of all nongenotyped animals. We used the sires from generations 3 and 4 as an illustration, but improvements in imputation quality must be observed for all other nongenotyped animals as well. This should also be relevant for the better imputation of genotypes of females with phenotypes. The effects of imputing genotypes of females in the context of enlarging a training set for 2 -step evaluations have been investigated by Pimentel et al. (2013) and Bouwman et al. (2014). Furthermore, genotypes of animals without phenotypes (i.e., which are just being predicted) influence the whole system and also their own prediction; this is also valid for validation studies. The choice of validation animals itself influences the whole system and can affect the predicted values of validation animals themselves, and thus the validation statistics.

The increase in the number of sons per sire shown in Table 2 illustrates the quantitative gain in information for imputation by adding genotypes of culled males. However, these additional genotypes should also result in a qualitative gain of information for imputation, especially in the presence of selection. Patry and Ducrocq (2011) stated that genomic preselection of young bulls

Table 2. Number of genotyped sons per sire from generation 3 to 4, calculated and expected correlation between imputed and true genotypes for different scenarios

\begin{tabular}{lllcc}
\hline Item & Scenario 1 & Scenario 2 & Scenario 3 & Scenario 4 \\
\hline Number of sons per sire & 2.41 & 3.10 & 14.51 & 2.63 \\
Calculated correlation (SD) & $0.77(0.06)$ & $0.82(0.06)$ & $0.93(0.06)$ & $0.82(0.07)$ \\
Expected correlation & 0.66 & 0.71 & 0.91 & 0.68 \\
\hline
\end{tabular}


should cause bias and loss of accuracy in subsequent BLUP evaluations of dairy cattle. Those authors reported that including genomic information from culled genotyped candidates in the BLUP evaluations removed bias and increased accuracy. Their study concerned 2-step systems, in which phenotypes for the first step are derived from the (potentially biased) BLUP evaluations. Nevertheless, genomic preselection should also affect 1-step evaluations if genotypes of culled males are neglected. Including their genotypes would account more properly for the Mendelian sampling information on which selection was based. Because they do not contribute any phenotypic information to the system, the way this information is accounted for most likely comes from the imputation of their ancestors, for which phenotypic information is available. So the improvement in imputation quality that we describe here is also the mechanism underlying the correction for the effects of genomic preselection in ssGBLUP; this would be part of the qualitative gain from additional genotypes of culled males. As mentioned, we observed similar trends across scenarios in the simulation without selection; therefore, most of the increase in reliabilities that we observed here was due to the quantitative gain from additional information in $\mathbf{G}$, as depicted in Table 2. Nevertheless, results from S4 (Figure 1) indicate at least some qualitative gains.

The magnitude of improvement in reliability observed in our study was useful to illustrate the mechanisms involved in the transmission of information from genotyped animals in the $\mathbf{H}$ matrix to the predicted breeding values. This order of magnitude was surely a consequence of the structure of the simulated population in which the reliabilities were low in the reference scenario (S1). Improvements in reliability from adding genotypes of culled animals would possibly be lower in real genomic breeding programs with large reference populations. However, the addition of genotypes from culled animals may be relevant in the case of small populations in which a limited amount of genotypic information is available (Andonov et al., 2017).

In conclusion, adding genotypes from culled animals in ssGBLUP leads to an improvement in prediction reliability. This improvement can be explained by the higher imputation accuracy of nongenotyped animals.

\section{ACKNOWLEDGMENTS}

We gratefully acknowledge the Arbeitsgemeinschaft Süddeutscher Rinderzucht- und Besamungsorganisationen e.V. (Munich, Germany) for their financial support within the research cooperation "Zukunftswege," and two anonymous reviewers for useful suggestions.

\section{REFERENCES}

Aguilar, I., I. Misztal, D. L. Johnson, A. Legarra, S. Tsuruta, and T. J. Lawlor. 2010. Hot topic: A unified approach to utilize phenotypic, full pedigree, and genomic information for genetic evaluation of Holstein final score. J. Dairy Sci. 93:743-752.

Andonov, S., D. A. L. Lourenco, B. O. Fragomeni, Y. Masuda, I. Pocrnic, S. Tsuruta, and I. Misztal. 2017. Accuracy of breeding values in small genotyped populations using different sources of external information - A simulation study. J. Dairy Sci. 100:395-401.

Bouwman, A. C., J. M. Hickey, M. P. L. Calus, and R. F. Veerkamp. 2014. Imputation of non-genotyped individuals based on genotyped relatives: Assessing the imputation accuracy of a real case scenario in dairy cattle. Genet. Sel. Evol. 46:6.

Christensen, O. F., and M. S. Lund. 2010. Genomic prediction when some animals are not genotyped. Genet. Sel. Evol. 42:2.

Fernando, R. L., J. C. M. Dekkers, and D. J. Garrick. 2014. A class of Bayesian methods to combine large numbers of genotyped and non-genotyped animals for whole-genome analyses. Genet. Sel. Evol. 46:50

Legarra, A., I. Aguilar, and I. Misztal. 2009. A relationship matrix including full pedigree and genomic information. J. Dairy Sci. 92:4656-4663.

Legarra, A., O. F. Christensen, I. Aguilar, and I. Misztal. 2014. Single Step, a general approach for genomic selection. Livest. Sci. 166:54-65.

Lidauer, M., K. Matilainen, E. Mäntysaari, T. Pitkänen, and M. Taskinen. 2015. MiX99: A software package for solving large mixed model equations. Release VIII/2015. Natural Resources Institute Finland (Luke), Jokioinen, Finland.

Lourenco, D. A. L., I. Misztal, S. Tsuruta, I. Aguilar, T. J. Lawlor, S. Forni, and J. I. Weller. 2014. Are evaluations on young genotyped animals benefiting from the past generations? J. Dairy Sci. 97:3930-3942.

Lush, J. L. 1931. The number of daughters necessary to prove a sire. J. Dairy Sci. 14:209-220.

Meuwissen, T. H., B. J. Hayes, and M. E. Goddard. 2001. Prediction of total genetic value using genome-wide dense marker maps. Genetics 157:1819-1829.

Misztal, I., A. Legarra, and I. Aguilar. 2014. Using recursion to compute the inverse of the genomic relationship matrix. J. Dairy Sci. 97:3943-3952.

Patry, C., and V. Ducrocq. 2011. Accounting for genomic pre-selection in national BLUP evaluations in dairy cattle. Genet. Sel. Evol. 43:30.

Pausch, H., B. Aigner, R. Emmerling, C. Edel, K.-U. Götz, and R. Fries. 2013. Imputation of high-density genotypes in the Fleckvieh cattle population. Genet. Sel. Evol. 45:3.

Pimentel, E. C. G., M. Wensch-Dorendorf, S. König, and H. H. Swalve. 2013. Enlarging a training set for genomic selection by imputation of un-genotyped animals in populations of varying genetic architecture. Genet. Sel. Evol. 45:12.

Plieschke, L., C. Edel, E. C. G. Pimentel, R. Emmerling, J. Bennewitz, and K.-U. Götz. 2016. Systematic genotyping of groups of cows to improve genomic estimated breeding values of selection candidates. Genet. Sel. Evol. 48:73.

Pszczola, M., H. A. Mulder, and M. P. L. Calus. 2011. Effect of enlarging the reference population with (un)genotyped animals on the accuracy of genomic selection in dairy cattle. J. Dairy Sci. 94:431-441.

Pszczola, M., T. Strabel, J. A. M. van Arendonk, and M. P. L. Calus. 2012. The impact of genotyping different groups of animals on accuracy when moving from traditional to genomic selection. J. Dairy Sci. 95:5412-5421.

Sargolzaei, M., and F. S. Schenkel. 2009. QMSim: A large-scale genome simulator for livestock. Bioinformatics 25:680-681.

VanRaden, P. M. 2008. Efficient methods to compute genomic predictions. J. Dairy Sci. 91:4414-4423. 